\title{
OPEN The impact of heat on kidney stone presentations in South Carolina under two climate change scenarios
}

\author{
Jason Kaufman ${ }^{1}$, Ana M. Vicedo-Cabrera ${ }^{2,3}$, Vicky Tam ${ }^{4}$, Lihai Song ${ }^{4}$, Ethan Coffel $^{5}$ \& \\ Gregory Tasian ${ }^{6,7} \bowtie$
}

The risk of kidney stone presentations increases after hot days, likely due to greater insensible water losses resulting in more concentrated urine and altered urinary flow. It is thus expected that higher temperatures from climate change will increase the global prevalence of kidney stones if no adaptation measures are put in place. This study aims to quantify the impact of heat on kidney stone presentations through 2089, using South Carolina as a model state. We used a time series analysis of historical kidney stone presentations (1997-2014) and distributed lag non-linear models to estimate the temperature dependence of kidney stone presentations, and then quantified the projected impact of climate change on future heat-related kidney stone presentations using daily projections of wetbulb temperatures to 2089 , assuming no adaptation or demographic changes. Two climate change models were considered-one assuming aggressive reduction in greenhouse gas emissions (RCP 4.5) and one representing uninibited greenhouse gas emissions (RCP 8.5). The estimated total statewide kidney stone presentations attributable to heat are projected to increase by $2.2 \%$ in RCP 4.5 and $3.9 \%$ in RCP 8.5 by $2085-89$ (vs. 2010-2014), with an associated total excess cost of $\sim \$ 57$ million and $\sim \$ 99$ million, respectively.

Kidney stone disease (nephrolithiasis) is a painful condition affecting roughly one in eleven Americans, the incidence of which has increased in the last 20 years, particularly among women and adolescents ${ }^{1,2}$. Kidney stone disease is a disorder of mineral metabolism that is punctuated by acute symptomatic events that are usually caused by movement of a stone to the ureter and may be recurrent over the lifetime. It is well established that high ambient temperatures increase the risk of developing kidney stone disease and presenting with acute, symptomatic stones ${ }^{3-5}$. The evidence in support of the relationship between ambient climate and kidney stone disease first arose from observations that kidney stone presentations increase in warmer months, and that there is a North to South increase in kidney stone incidence in the United States ${ }^{6}$. More recently, our group and others have more precisely defined the relationship between hot days and kidney stone presentations, which is characterized by a short lag of up to 2 days and heterogeneity by geographic region and sex ${ }^{5,7,8}$. One proposed mechanism is that higher evaporative water losses leads to more concentrated urine, creating an environment in which crystallization of calcium, oxalate, uric acid, and phosphate is more likely ${ }^{9,10}$. This exact mechanism, however, has not been elucidated.

As global ambient temperatures increase from climate change, it is expected that the prevalence of kidney stone disease and associated acute kidney stone presentations will follow. The current study aims to quantify the attribute risk and associated cost of kidney stone presentations as a function of heat and humidity under two scenarios of climate change in South Carolina. By modeling two scenarios of future climate change-one more aggressive, one more conservative-we can also compare how the burden of kidney stone disease as reflected by acute presentations may be affected by climate policy.

\footnotetext{
${ }^{1}$ Perelman School of Medicine at the University of Pennsylvania, Philadelphia, PA, USA. ${ }^{2}$ Institute of Social and Preventive Medicine, University of Bern, Bern, Switzerland. ${ }^{3}$ Oeschger Center for Climate Change Research, University of Bern, Bern, Switzerland. ${ }^{4}$ Data Science and Biostatistics Unit, Department of Biomedical and Health Informatics, Children's Hospital of Philadelphia, Philadelphia, PA, USA. ${ }^{5}$ Department of Geography and the Environment, Syracuse University, Syracuse, NY, USA. ${ }^{6}$ Division of Pediatric Urology, Children's Hospital of Philadelphia, Philadelphia, PA, USA. 'Departments of Surgery and Biostatistics, Epidemiology, and Informatics, Perelman School of Medicine at the University of Pennsylvania, Philadelphia, PA, USA. ${ }^{\circledR}$ email: tasiang@chop.edu
} 


\section{Methods}

Study design. We performed a two-step study to project the impact of heat on future kidney stone presentations in South Carolina. As a model state, South Carolina offers insight into the effect that climate change will have on a region with an already high disease burden. South Carolina lies within the "kidney stone belt" of the Southeast US, a region with a higher incidence of kidney stone disease thought to be secondary to both diet and higher temperatures ${ }^{11}$. First, we performed an aggregated case-crossover study to determine the relationship between historic daily state-wide mean wet-bulb temperatures (WBT) and kidney stone presentations in South Carolina from 1997 to 2014 (adapted from Vicedo-Cabrera et al. ${ }^{8}$ ). Next, we projected the heat-related number of kidney stone presentations and associated cost to 2089 based on projected daily WBT under two climate change scenarios.

Exposure. The exposure used in this study is daily mean WBT, a moist heat metric that accounts for both ambient heat and humidity. We previously reported that wet-bulb temperature is the most accurate temperature metric for predicting kidney stone presentations in South Carolina ${ }^{12}$. The reason for this higher performance is thought to be due to the opposing effect of temperature and humidity on insensible water loss. Insensible water losses increase in higher temperatures, but decrease in higher relative humidities ${ }^{6,12,13}$. Observed daily mean WBT was obtained by averaging county-level measures of WBT across South Carolina from the NASA Land Data Assimilation Systems from January 1, 1997 to December 31, 2014 12,14.

Projections of future WBT were generated by Dr. Ethan Coffel, Department of Geography and the Environment at Syracuse University ${ }^{15}$. Six predictive datasets were considered: three general circulation models (GCMs) (Access 1-0, BCC-esm, and CNRM-cm5) forced by two representative concentration pathways (RCPs) of global climate change (RCP 4.5 and RCP 8.5). The GCMs were selected to capture some variability within each RCP, and were selected in consultation with the climate scientist on the team.

The RCPs represent different pathways of future climate change, depending on human factors such as land use change and efforts to mitigate climate change. RCP 4.5 and 8.5 lead to radiative forcing outcomes of 4.5 and $8.5 \mathrm{~W} / \mathrm{m}^{2}$ by 2100 , respectively, with higher values representing a greater greenhouse gas effect. GCMs project different trajectories in approaching that endpoint based on climatic factors including the atmosphere, oceans, and daily variations in weather ${ }^{16}$. Three GCMs were selected at random to capture a range of assumptions regarding day to day variation within each RCP, in consultation with the team's climate scientist. Each projection dataset was calibrated to minimize long-term differences between historic and projection datasets, using methodology adapted from Hempel et al. ${ }^{17}$.

RCP 4.5 represents an "intermediate" future, with shifts to lower-emissions sources of energy, the use of carbon capture technology, prices on $\mathrm{CO}_{2}$ emissions, and an expansion of forest lands ${ }^{16,18}$. RCP 8.5, conversely, represents a future with high population growth, high energy demand, little advancement towards greater efficiency, and a greater dependence on domestic, more easily accessible energy sources like coal which have a higher greenhouse gas footprint ${ }^{19}$. Under RCP 8.5, forest lands decrease and there are far fewer assumed policies to mitigate greenhouse gas emissions ${ }^{19}$. While RCP 4.5 represents more aggressive greenhouse gas mitigation strategies, RCP 8.5 represents a future with mostly uninhibited greenhouse gas emissions ${ }^{16}$. Comparing these two possible futures in this study, we can compare the projected effect that aggressive global climate policies, actions, or inactions could have on kidney stone presentations.

As the projection dataset covered the entire globe with a $2^{\circ} \times 2^{\circ}$ grid, we divided South Carolina into five geographic regions (Supplementary Appendix A), and averaged the daily WBT at the center of these regions to generate a single future daily mean WBT projection for the state per projection.

Outcome. The outcome was the number of index kidney stone presentations in South Carolina emergency rooms. For the first stage in which the temperature dependence of kidney stone presentations was modeled, the counts of kidney stone presentations from January 1, 1997 to December 31, 2014 was obtained from the South Carolina Medical Encounter Data and Financial Reports all-payer claims database. This database includes all emergency department visits, surgical procedures, and hospital admissions across South Carolina, with individual IDs and accompanying demographic data. Audits are regularly performed on this dataset to ensure $99.9 \%$ accuracy of diagnostic codes and $99.5 \%$ completion $^{20}$. Kidney stone cases were identified as emergency room visits associated with a primary International Classification of Diseases, 9th and 10th Revision codes for kidney and urinary tract stones (ICD-9 codes 592.0, 592.1, 592.9, and 274.11; ICD-10 N20). For individuals with multiple encounters with these codes, only the earliest encounter was considered so as to not double-count multiple encounters related to a single kidney stone event. Cost per presentation was simplified as the mean total charges incurred by all patients presenting through the emergency room with a primary presentation of kidney stone disease from January 1, 1997 to December 31, 2014, without adjusting for inflation. This average cost per patient between within these dates was $\$ 9525.95$.

Statistical analysis. The first step consisted of the estimation of the exposure-response curve between daily WBT and kidney stone presentations using historical data and the method and modelling specifications described in Vicedo-Cabrera et al. Briefly, conditional quasi-Poisson regression with distributed lag non-linear models (DLNMs) was used to determine the non-linear and delayed association accounting up to 10 days after the exposure. We fitted a natural spline with two internal knots equally spaced in the log scale in the exposurelag dimension. For the exposure-response dimension, we used a natural spline function, instead of the original quadratic b-spline, with internal knots at the 50th and 90th percentile of the temperature distribution. The use of natural splines allowed for the log-linear extrapolation of risk beyond the observed range of temperatures in 


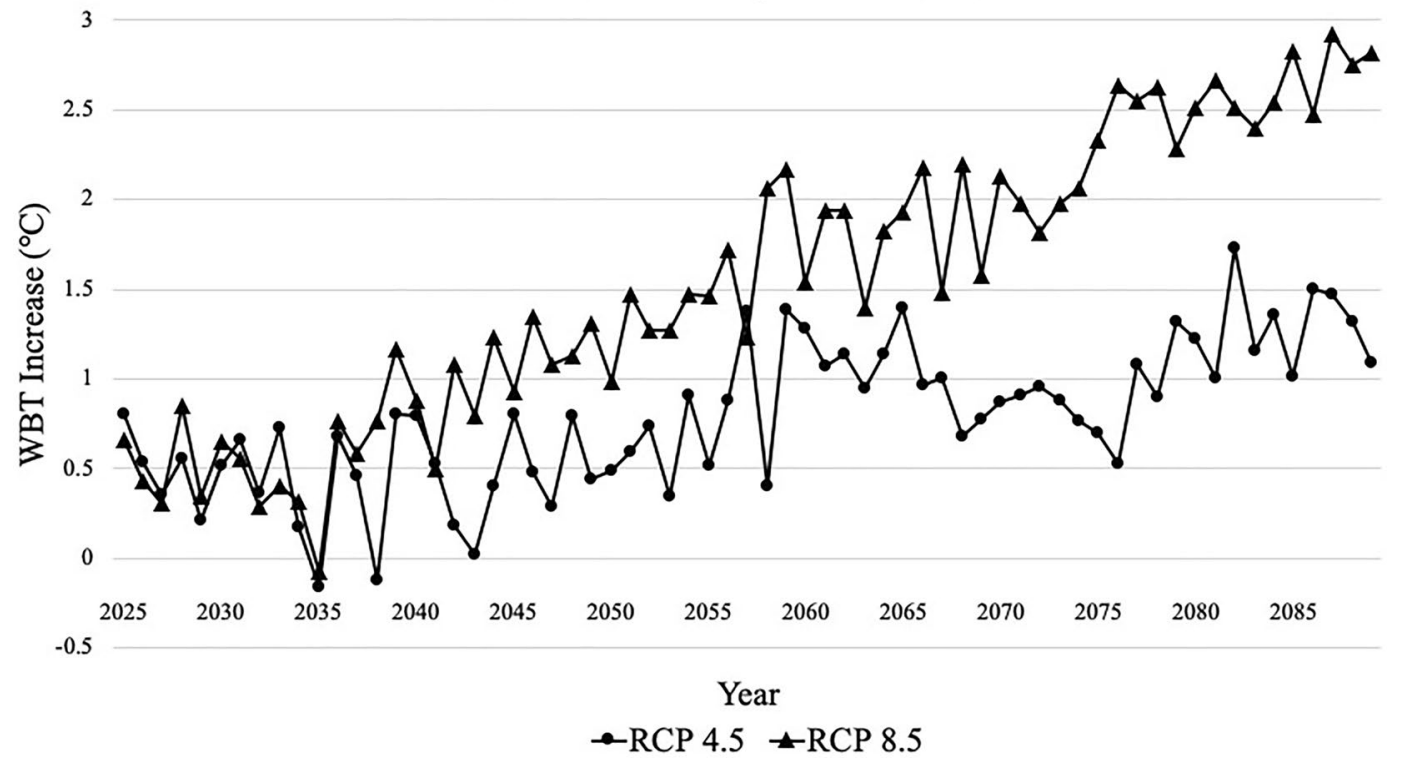

Figure 1. Increase in average annual WBT above average WBT between 1997 and 2014 for projected years in RCP 4.5 and RCP 8.5. Higher future WBT are associated with RCP 8.5 compared to RCP 4.5, due to greater projected future greenhouse gas concentration.

\begin{tabular}{|l|l|l|}
\hline Historic year range & Mean daily WBT $\left({ }^{\circ} \mathrm{C}\right)(\mathrm{IQR})$ & Maximum daily mean WBT $\left({ }^{\circ} \mathrm{C}\right)$ \\
\hline $1997-1999$ & $14.2(8.4-20.6)$ & 25.7 \\
\hline $2000-2004$ & $14.0(8.0-21.0)$ & 24.7 \\
\hline $2005-2009$ & $14.0(8.1-20.6)$ & 26.0 \\
\hline $2010-2014$ & $14.0(7.6-20.9)$ & 25.3 \\
\hline
\end{tabular}

Table 1. Mean and maximum daily WBT for historic periods assessed in this study.

the historical dataset, a critical consideration when projecting into years with temperatures higher than those ever observed in the historical period ${ }^{21}$.

In the second step, the overall cumulative exposure-response association was used to estimate the number of WBT-related kidney stone presentations in future periods using each of the six daily projections of WBT. The method used is described in detail in Vicedo-Cabrera et al. $^{21}$. The total number of kidney stone presentations attributed to heat in each 5-year period between 2025 and 2089 (above the mean from 2010 to 2014) was computed by summing the daily temperature-related number of cases on days with WBT $>6.7^{\circ} \mathrm{C}$ (defined as the temperature of minimum risk estimated from the exposure-response curve) (see Vicedo-Cabrera et al. ${ }^{21}$ ). Estimates were reported as average ensemble estimates across the 3 GCMs for each RCP. This modeling approach assumes no changes in population demographics or vulnerability (i.e., adaptation). That is, estimates reflect the impact on today's population in South Carolina if exposed to a warmer climate consistent with these two climate change scenarios. As measures of uncertainty, $95 \%$ empirical confidence intervals (eCI) were estimated using Monte Carlo simulations with 1000 samples of coefficients of the association over each of the GCM-specific series, assuming a normal distribution of the estimates.

\section{Results}

Both RCP 4.5 and 8.5 project an increase in temperatures from the 2010 s to the 2080 s, with RCP 8.5 projecting a larger increase in temperatures secondary to greater greenhouse gas emissions compared to RCP 4.5 (Fig. 1). Tables 1 and 2 describe characteristics of the historic and projected temperature datasets for both RCPs. RCP 4.5 projects a $2.3^{\circ} \mathrm{C}$ increase in mean temperature per 5-year period from 2010-2014 to 2085-2089, while RCP 8.5 projects a $3.6^{\circ} \mathrm{C}$ increase in the same time frame (Table 2). The number of days exceeding the historic maximum daily mean from 1997 to $2014\left(26.0^{\circ} \mathrm{C}\right)$ are also expected to increase over the course of the century, with far more days exceeding this historic maximum in RCP 8.5 (Table 2).

The risk of kidney stone presentations increases non-linearly during the 10 days following a daily WBT greater than the referent temperature of $6.7^{\circ} \mathrm{C}$ with no evidence of a ceiling effect at higher temperatures (Fig. 2a). Risk increases with higher temperatures and is greatest in the 2 days following a temperature event (Fig. 2b). The increase in heat-related kidney stone events (above the baseline from 2010 to 2014) from 2025 to 2089 is 5938 


\begin{tabular}{|c|c|c|c|c|c|c|}
\hline \multirow[b]{2}{*}{ Projected year range } & \multicolumn{3}{|l|}{ RCP 4.5} & \multicolumn{3}{|l|}{ RCP 8.5} \\
\hline & $\begin{array}{l}\text { Mean daily WBT }\left({ }^{\circ} \mathrm{C}\right) \\
(\mathrm{IQR})\end{array}$ & \# Days above $26.0^{\circ} \mathrm{C}$ & \# Days above $6.7^{\circ} \mathrm{C}$ & $\begin{array}{l}\text { Mean daily WBT }\left({ }^{\circ} \mathrm{C}\right) \\
\text { (IQR) }\end{array}$ & \# Days above $26.0^{\circ} \mathrm{C}$ & \# Days above $6.7^{\circ} \mathrm{C}$ \\
\hline 2025-2029 & $14.5(8.7-21.2)$ & 0 & 1525 & $14.5(8.8-21.1)$ & 0 & 1550 \\
\hline $2030-2034$ & $14.5(8.7-21.1)$ & 0 & 1526 & $14.4(8.9-21.1)$ & 0 & 1543 \\
\hline $2035-2039$ & $14.3(8.3-21.3)$ & 0 & 1490 & $14.6(8.5-21.6)$ & 0 & 1515 \\
\hline $2040-2044$ & $14.4(8.3-21.3)$ & 0 & 1496 & $14.9(8.9-21.5)$ & 0 & 1572 \\
\hline 2045-2049 & $14.6(8.5-21.6)$ & 0 & 1527 & $15.2(9.6-21.9)$ & 0 & 1586 \\
\hline $2050-2054$ & $14.6(8.6-21.6)$ & 0 & 1536 & $15.3(9.3-22.1)$ & 0 & 1591 \\
\hline $2055-2059$ & $14.9(9.1-22.0)$ & 0 & 1565 & $15.7(10.1-22.6)$ & 4 & 1624 \\
\hline $2060-2064$ & $15.1(9.0-21.9)$ & 0 & 1565 & $15.7(9.9-22.6)$ & 5 & 1620 \\
\hline $2065-2069$ & $15.0(9.1-21.7)$ & 0 & 1564 & $15.9(9.5-22.7)$ & 8 & 1642 \\
\hline $2070-2074$ & $14.9(8.8-22.0)$ & 0 & 1576 & $16.0(10.2-22.7)$ & 7 & 1664 \\
\hline 2075-2079 & $14.9(8.6-22.2)$ & 0 & 1516 & $16.5(10.6-23.2)$ & 23 & 1703 \\
\hline $2080-2084$ & $15.3(9.2-22.4)$ & 0 & 1594 & $16.5(10.7-23.3)$ & 46 & 1687 \\
\hline $2085-2089$ & $15.3(9.3-22.2)$ & 0 & 1576 & $16.8(10.7-23.8)$ & 69 & 1665 \\
\hline
\end{tabular}

Table 2. Projected mean WBT and days exceeding historic maximum $\left(26.0^{\circ} \mathrm{C}\right)$ and the referent temperature $\left(6.7^{\circ} \mathrm{C}\right)$ in each projected 5 -year period.

a Overall exposure-response association

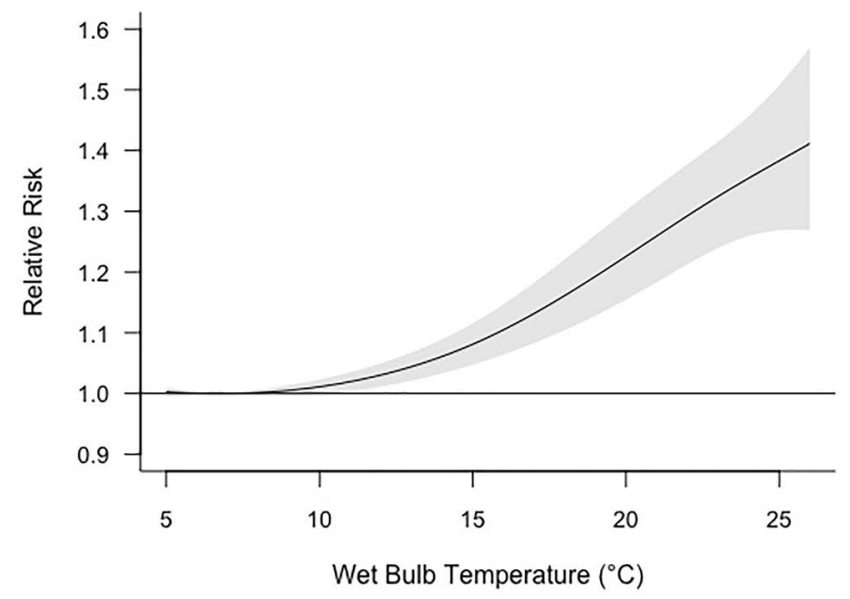

b Lag-response at 99th pct (vs. reference)

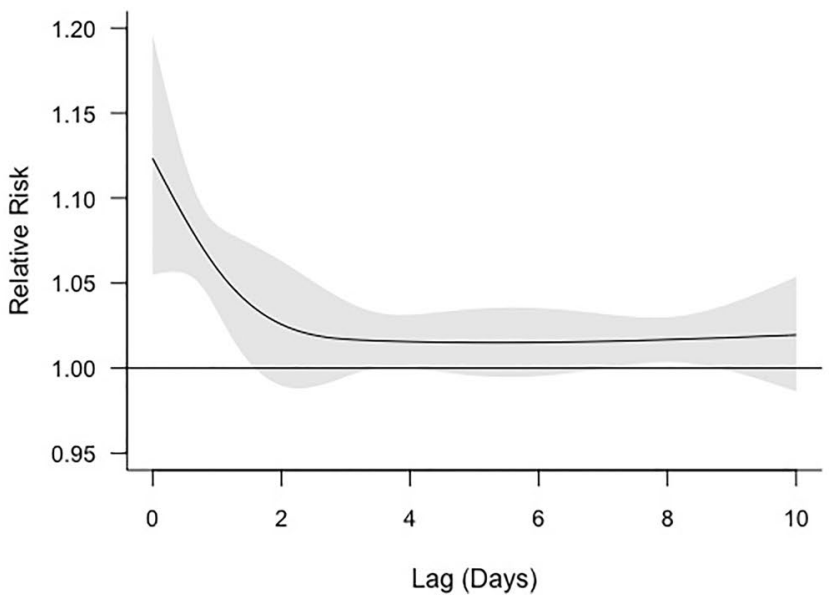

Figure 2. (a) The overall cumulative exposure-response relationship between daily WBT cumulated over 10 -day lag period and kidney stone presentations, relative to $6.7^{\circ} \mathrm{C}$. (b) The lag-response relationship shows the distribution of risk over 10 days following a daily WBT at the 99 th percentile, relative to the referent temperature of $6.7^{\circ} \mathrm{C}$.

(95\% CI 3730-9418) for RCP 4.5 and 10,431 (95\% CI 6724-15,581) for RCP 8.5 (Table 3). The total cost attributable to these excess kidney stones from $2025-2089$ is $\$ 56,565,091$ for RCP 4.5 and $\$ 99,365,184$ for RCP 8.5 (Table 3). The increase in the proportion of heat-related kidney stone presentations per 5 year period above the $2010-2014$ baseline is $0.62 \%$ and $0.77 \%$ in the $2025-2029$ period and $2.2 \%$ and $3.9 \%$ in the $2085-2089$ period (RCP 4.5 and 8.5 , respectively).

\section{Discussion}

This analysis generated a projection of emergent kidney stone presentations and related costs in South Carolina under two different scenarios of climate change, one scenario with high future greenhouse gas emissions (RCP 8.5) and one path assuming more aggressive future climate mitigation policies (RCP 4.5). This analysis suggests a total increase of 5938 emergent kidney stone presentations attributed to heat with an associated total cost of $\sim \$ 57$ million from 2025 to 2089 in RCP 4.5, compared to a total projected increase of 10,431 emergent kidney stone presentations attributed to heat and a total cost of $\sim \$ 99$ million from 2025 to 2089 in RCP 8.5. While it is impossible to predict with certainty how future policies will slow or hasten greenhouse gas emission and anthropogenic climate change, and to know exactly what future daily temperatures will be, this analysis suggests that an increased burden of kidney stone disease on healthcare systems attributed to climate warming 


\begin{tabular}{|c|c|c|c|c|}
\hline \multirow[b]{2}{*}{ Years } & \multicolumn{2}{|l|}{ RCP 4.5} & \multicolumn{2}{|l|}{ RCP 8.5} \\
\hline & $\begin{array}{l}\text { Increase in heat-related kidney stones } \\
\text { presentations }(95 \% \mathrm{CI})\end{array}$ & Associated excess charges & $\begin{array}{l}\text { Increase in heat-related kidney stones } \\
\text { presentations }(95 \% \mathrm{CI})\end{array}$ & Associated excess charges \\
\hline $2025-2029$ & $212(61-396)$ & $\$ 2,019,501.40$ & $258(128-443)$ & $\$ 2,457,695.10$ \\
\hline $2030-2034$ & $316(179-450)$ & $\$ 3,010,200.20$ & $348(94-673)$ & $\$ 3,315,030.60$ \\
\hline $2035-2039$ & $284(154-457)$ & $\$ 2,705,369.80$ & $250(133-436)$ & $\$ 2,381,487.50$ \\
\hline $2040-2044$ & $416(270-565)$ & $\$ 3,962,795.20$ & $548(285-849)$ & $\$ 5,220,220.60$ \\
\hline $2045-2049$ & $305(94-514)$ & $\$ 2,905,414.75$ & $541(301-853)$ & $\$ 5,153,538.95$ \\
\hline $2050-2054$ & $341(181-522)$ & $\$ 3,248,348.95$ & $695(413-1078)$ & $\$ 6,620,535.25$ \\
\hline $2055-2059$ & $433(192-782)$ & $\$ 4,124,736.35$ & $801(401-1290)$ & $\$ 7,630,285.95$ \\
\hline $2060-2064$ & $601(368-874)$ & $\$ 5,725,095.95$ & $1011(553-1674)$ & $\$ 9,630,735.45$ \\
\hline $2065-2069$ & $607(236-1025)$ & $\$ 5,782,251.65$ & $1010(597-1521)$ & $\$ 9,621,209.50$ \\
\hline $2070-2074$ & $523(245-1008)$ & $\$ 4,982,071.85$ & $1102(546-1846)$ & $\$ 10,497,596.90$ \\
\hline $2075-2079$ & $499(229-800)$ & $\$ 4,753,449.05$ & $1110(558-1854)$ & $\$ 10,573,804.50$ \\
\hline $2080-2084$ & $615(217-1094)$ & $\$ 5,858,459.25$ & $1348(720-2200)$ & $\$ 12,840,980.60$ \\
\hline $2085-2089$ & $786(289-1448)$ & $\$ 7,487,396.70$ & $1409(717-2256)$ & $\$ 13,422,063.55$ \\
\hline
\end{tabular}

Table 3. Increase in the number of kidney stones attributed to heat (defined as WBT above $6.7^{\circ} \mathrm{C}$ ) and associated charges projected under RCP 4.5 and RCP 8.5 by 5 -year interval between 2025 and 2089 , versus 2010-2014 baseline.

is very likely. This analysis additionally demonstrates how much of that increased burden could be curbed with moderately aggressive climate action.

A landmark 2008 study by Brikowski, et al. estimated that the number of Americans living in a "high risk" zone for kidney stones will increase from $40 \%$ in 2000 to $56 \%$ in 2050 . Since 2008 , we have a greater understanding of the temperature dependence of nephrolithiasis, which allows for more precise estimates of the impact of climate change on kidney stone presentations. While the Brikowski study applied hypothesized linear and non-linear relationships between temperature and kidney stone disease to the entire US, it has since been demonstrated that the relationship between temperature and kidney stone disease varies by geographic location, and that neither the hypothesized linear nor non-linear models accurately reflect the true exposure risk curve ${ }^{5}$. In addition, it has been demonstrated that moist-heat metrics such as WBT model the relationship between climate and kidney stone presentation better than dry bulb temperature alone, with or without corrections for relative humidity ${ }^{5,8}$, as temperature and humidity contribute differently to the extent of evaporative water loss ${ }^{12}$. Though more limited in geographic scope, the current study improves on this seminal 2008 study in several ways. In this study, we empirically defined the precise relationship between heat, humidity, and kidney stone presentation using historic data congruent with the study location. We also used WBT projections that account for future day-to-day variation in heat and humidity to define and project kidney stone disease risk.

There are several important assumptions to consider when interpreting the results of this study. First, we assumed a constant population at risk of kidney stone events from year to year; in reality, South Carolina's population will change over time. While population increase is likely (and thus our estimates may be an underestimate), it is also likely that population will adapt better as hot and humid days become more common, e.g. by spending more time in climate-controlled environments and drinking more fluids. We chose not to consider adaptation as efforts accurately model this adaptation are often overly simplistic ${ }^{21,22}$. In addition, the future effect of adaptation would likely vary by population characteristics and has not been well quantified ${ }^{23}$. We also applied a single risk curve for the entire population although the risk of temperature related kidney stone presentations is sexually dimorphic ${ }^{8}$. It is likely, however, that our population-wide estimates are accurate assuming a consistent population-wide distribution between males and females. Finally, South Carolina has recently experienced an observed rapid increased incidence in kidney stone disease, with a $\sim 1 \%$ increase in mean annual incidence of kidney stone disease from 1997 to 2012, and a particularly rapid rise among adolescents ${ }^{2}$. We did not project future increases in non-heat related presentations given the uncertainty of future shifts in the epidemiology of the disease.

Limitations to this study include the uncertain causal inference in the temperature-dependence of kidney stone presentations. Second, our estimates of total costs were based only on the medical costs incurred by a patient at their emergency department visit with a kidney stone. We did not consider inflation or indirect costs such as loss of work. Thus, the actual "cost" to society of the increased incidence of kidney stone disease are likely far greater. Third, we only considered one geographic area. Our group previously reported that the shape of the exposure response curve differs by location. These results, therefore, should not be extrapolated to other parts of the United States or other areas of the globe. As more data on kidney stone presentations and other heat-related health outcomes become available, these projections will be possible at a larger scale.

Despite these limitations, our analysis is a model to conceptualize how the burden of kidney stone disease is expected to progress with climate change, and also how mitigations to greenhouse gas emissions can offset some of this burden. With climate change a modern reality, the US can expect warmer days overall and more frequent extreme heat events ${ }^{24}$. A recent analysis of the proposed resolutions at the 2021 United Climate Change 
Conference indicated a likely increase in mean global temperature to below $2{ }^{\circ} \mathrm{C}$ by 2100 with adherence to these resolutions ${ }^{25}$, an outcome just below the RCP 4.5 pathway represented in this study. As our analysis shows, under even a relatively conservative projection of climate change we can expect a higher incidence and cost of symptomatic kidney stones, particularly in the near future before any compensatory adaptive efforts.

As the research community continues to elucidate the impacts that climate change will have on the environment and human well-being, it is important to explore the burden of climate change on human health via physiologic stressors. Improving our understanding of this relationship will help health systems better prepare and encourage the public and policymakers to prioritize sustainable behaviors and policies to mitigate climate change.

Received: 16 June 2021; Accepted: 13 December 2021

Published online: 10 January 2022

\section{References}

1. Scales, C. D. et al. Urinary stone disease: Advancing knowledge, patient care, and population health. Clin. J. Am. Soc. Nephrol. 11, 1305-1312 (2016).

2. Tasian, G. E. et al. Annual incidence of nephrolithiasis among children and adults in South Carolina from 1997 to 2012. Clin. J. Am. Soc. Nephrol. 11, 488-496 (2016).

3. Soucie, J. M., Thun, M. J., Coates, R. J., McClellan, W. \& Austin, H. Demographic and geographic variability of kidney stones in the United States. Kidney Int. 46, 893-899 (1994).

4. Brikowski, T. H., Lotan, Y. \& Pearle, M. S. Climate-related increase in the prevalence of urolithiasis in the United States. PNAS 105, 9841-9846 (2008).

5. Tasian, G. E. et al. Daily mean temperature and clinical kidney stone presentation in five US metropolitan areas: A time-series analysis. Environ. Health Persp. 122, 1081-1087 (2014).

6. Fakheri, R. J. \& Goldfarb, D. S. Ambient temperature as a contributor to kidney stone formation: Implications of global warming. Kidney Int. 79(11), 1178-1185 (2011).

7. Chi, B. H. et al. Daily mean temperature and urolithiasis presentation in six cities in Korea: Time-series analysis. J. Korean Med. Sci. 32(6), 999-1008 (2017).

8. Vicedo-Cabrera, A. M., Goldfarb, D. S., Kopp, R. E., Song, L. \& Tasian, G. E. Sex differences in the temperature dependence of kidney stone presentations: A population-based aggregated case-crossover study. Urolithiasis 48, 37-46 (2020).

9. Masterson, J. H. et al. Changes in urine parameters after desert exposure: Assessment of stone risk in United States Marines transiently exposed to a desert environment. J. Urol. 189, 165-170 (2013).

10. Eisner, B. H. et al. The effects of ambient temperature, humidity and season of year on urine composition in patients with nephrolithiasis. BJU Int. 110, E1014-E1017 (2012).

11. Sas, D. J., Hulsey, T. C., Shatat, I. F. \& Orak, J. K. Increasing incidence of kidney stones in children evaluated in the emergency department. J. Pediatr. 157, 132-137 (2010).

12. Ross, M. E. et al. Assessment of the combination of temperature and relative humidity on kidney stone presentations. Environ. Res. 162, 97-105 (2018).

13. Budd, G. M. Wet-bulb globe temperature (WBGT) -Its history and its limitations. J. Sci. Med. Sport 11, 20-32 (2008).

14. Mitchell, K. E. et al. The multi-institution North American Land Data Assimilation System (NLDAS): Utilizing multiple GCIP products and partners in a continental distributed hydrological modeling system. J. Geophys. Res. Atmos. https://doi.org/10.1029/ 2003JD003823 (2004).

15. Coffel, E. D., Horton, R. M. \& de Sherbinin, A. Temperature and humidity based projections of a rapid rise in global heat stress exposure during the 21st century. Environ. Res. Lett. 13, 014001 (2018).

16. Pachauri, R. K. \& Meyer, L. A. (eds) IPCC, 2014: Climate Change 2014: Synthesis Report. Contribution of Working Groups I, II and III to the Fifth Assessment Report of the Intergovernmental Panel on Climate Change 151 (IPCC, 2014).

17. Hempel, S., Frieler, K., Warszawski, L., Schewe, J. \& Piontek, F. A trend-preserving bias correction-The ISI-MIP approach. Earth Syst. Dyn. 4, 219-236 (2013).

18. Thomson, A. M. et al. RCP4.5: A pathway for stabilization of radiative forcing by 2100. Clim. Change 109(1-2), 77 (2011).

19. Riahi, K. et al. RCP 8.5-A scenario of comparatively high greenhouse gas emissions. Clim. Change 109, 33 (2011).

20. South Carolina Data Oversight Council. Principles and Protocol for the Release of Health Care Data. South Carolina State Documents Depository (South Carolina Data Oversight Council, 2014).

21. Vicedo-Cabrera, A. M., Sera, F. \& Gasparrini, A. Hands-on tutorial on a modeling framework for projections of climate change impacts on health. Epidemiology 30, 321-329 (2019).

22. Gosling, S. N. et al. Adaptation to climate change: A comparative analysis of modeling methods for heat-related mortality. Environ. Health Persp. 125, 087008 (2017).

23. Morefield, P. E., Fann, N., Grambsch, A., Raich, W. \& Weaver, C. Heat-related health impacts under scenarios of climate and population change. Int. J. Environ. Res. 15, 2438 (2014).

24. Luber, G. \& McGeehin, M. Climate change and extreme heat events. Am. J. Prev. Med. 35, 429-435 (2008).

25. Ou, Y. et al. Can updated climate pledges limit warming well below $2{ }^{\circ} \mathrm{C}$ ? Science 374(6568), 693-695 (2021).

\section{Author contributions}

J.K. wrote the main manuscript text, while all authors contributed to and reviewed the manuscript. J.K. adapted the code provided by A.V.-C. to apply to the specific datasets used in this study. A.V.-C. guided the analytical framework and provided and troubleshooted the code that generated all figures and results. V.T. guided the geographic analyses to generate the daily temperature projections that covered the state of South Carolina from global temperature projection dataset. L.S. helped identify and work with key datasets, particularly the South Carolina health dataset. E.C. provided datasets of projections of daily wet bulb temperature under multiple climate scenarios across the entire globe, and guided the selection of the climate scenarios used in this study. G.T. guided the design and implementation of the project, and built the team capable of achieving the project's goals.

\section{Funding}

Funding was provided by the American Urological Association Foundation and also by the National Institute of Diabetes and Digestive and Kidney Diseases (K23DK106428). 


\section{Competing interests}

The authors declare no competing interests.

\section{Additional information}

Supplementary Information The online version contains supplementary material available at https://doi.org/ 10.1038/s41598-021-04251-2.

Correspondence and requests for materials should be addressed to G.T.

Reprints and permissions information is available at www.nature.com/reprints.

Publisher's note Springer Nature remains neutral with regard to jurisdictional claims in published maps and institutional affiliations.

(c) (1) Open Access This article is licensed under a Creative Commons Attribution 4.0 International License, which permits use, sharing, adaptation, distribution and reproduction in any medium or format, as long as you give appropriate credit to the original author(s) and the source, provide a link to the Creative Commons licence, and indicate if changes were made. The images or other third party material in this article are included in the article's Creative Commons licence, unless indicated otherwise in a credit line to the material. If material is not included in the article's Creative Commons licence and your intended use is not permitted by statutory regulation or exceeds the permitted use, you will need to obtain permission directly from the copyright holder. To view a copy of this licence, visit http://creativecommons.org/licenses/by/4.0/.

(c) The Author(s) 2022 